\title{
Fast and High-performance Multi-convolution Deep Neural Network Structure with Residuals
}

\author{
Yunpeng Wang \\ Department of Electrical and \\ Computer Engineering \\ Yokohama National University \\ Japan 240-8501 \\ Email: wang-yunpeng-sr@ynu.jp
}

\author{
Yasutaka Fujimoto \\ Department of Electrical and \\ Computer Engineering \\ Yokohama National University \\ Japan 240-8501 \\ Email: fujimoto@ynu.ac.jp
}

\begin{abstract}
Very deep convolution neural networks show a great improvement over competitive benchmarks. But the depth also brings extremely high computational cost. In this paper, inspired by Inception module, we introduce a new convolutional neural network module that combines residual structure and multiple convolution. A residual structure is mainly adopted to solve the gradient vanishing problem. And unlike the concatenation in the inception module, multiple convolution is used to find the most proper feature maps through self-optimizing training. With this module, we no longer need to carefully optimize the convolution structure of network, but can attain state-of-the-art results on CIFAR-10, MNIST and CIFAR-100 with 26 layers and only 19k parameters.
\end{abstract}

\section{INTRODUCTION}

Convolutional neural networks have become popular since the booming of hardware and GPU computation ability and have been inspired by AlexNet [1] in 2009. Arguably, convolutional neural networks are going deeper, from only five convolutional layers in AlexNet [1] to the VGG network [2] with 19 layers and the Inception network [3] with 22 layers, notably the Residual Network has 152 layers [4].

Network depth can highly influence the expressiveness of a model [5]. However, very deep neural networks also cause some new challenges, such as: the vanishing gradients problem in backward propagation and a very long training time.

The vanishing gradients problem is also a big challenge in optimizing a neural network. For the back propagation method, the updating of weights is done by multiplication of the partial derivative, however this value becomes extremely small in the lower layers based on chain rules which transforms the lower layers into dead zones without being updated. Several methods have been proposed to solve this problem, such as batch normalization [6] and supervision of hidden layers [7].

Moreover, long training time is a serious problem when a network becomes very deep. Even with multiple state-ofthe-art GPUs, CPUs and Cuda acceleration, models like the 152-layer ResNet still require several weeks to converge on the ImageNet dataset [4].

The goal of this work is to reach a better performance with as few layers and parameters as we can. In many domains, high computational speed is the most important characteristic for the whole system. Besides, optimizing the size of kernels is also a tough work for a neural network. Slight changes in the kernel sizes of different layers can considerably affect the final performance.

In this paper, we propose multiple convolutional layers with residuals. Results of our model surpass ResNet [4] in several competitive benchmarks with less than one fifth of the layers of ResNet [4]. With this structure, we finally obtained 94.33\% accuracy on CIFAR-10, 99.69\% accuracy on MNIST and $74.74 \%$ on CIFAR-100.

\section{BACKGROUND AND RELATED WORK}

Many researches are focusing on training very deep neural networks. Earlier works have attempted tried to alleviate the vanishing gradient problem by adopting a greedy layerwise training, pre-training or better initialization [8,9,10].A remarkable recent contribution to train very deep networks is batch normalization [6], which standardizes the mean and variance of the hidden layers with two additional trainable parameters. This approach reduces the vanishing gradients problem and achieves a better regularizing effect.

Residual networks, known as ResNets [4], simplify highway networks using a shortcut based on identity mapping. This method not only improves the training efficiency, but also presents improved feature reuse. The authors think that the networks become worse at function approximation because the gradients and training signals vanish owing to backpropagation through many layers. To fix these issues, they propose to add a shortcut connection to the output of convolution. Formally, if $y_{l}$ denotes the output of $l^{t h}$ layer and $f(\cdot)$ denotes typical convolutional transformation from layer $l-1$ to layer $l$, we have

$$
y_{l}=\operatorname{Re} L U\left(f\left(y_{l-1}\right)+y_{l-1}\right)
$$

where the second $y_{l-1}$ is identity transformation and $R e L U$ represents activation function [11]. Fig. 1 shows an example of function $f(\cdot)$ with two convolution layers and batch normalization layers. When the dimensions of the output $f\left(y_{l-1}\right)$ does not match those of the input $y_{l-1}$, the authors use $1 \times 1$ convolution layer as a linear transformation to change the dimensions of $y_{l-1}$ to match those of the output. Based on 


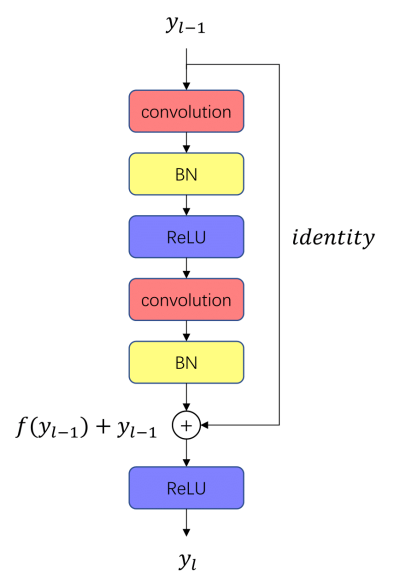

Fig. 1. ResNet structure at $l^{\text {th }}$ layer

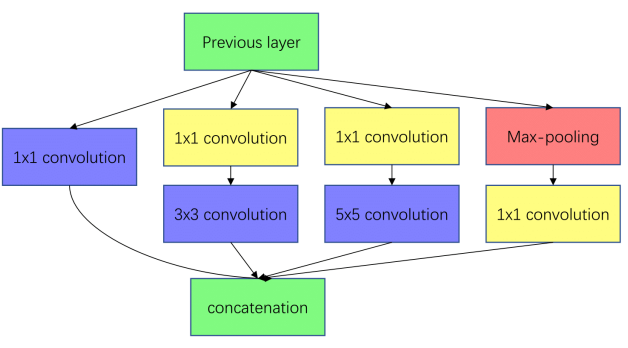

Fig. 2. A close look at Inception module

function (1), gradients and features can pass back and forth between the layers via identity mapping and thereby the issue of vanishing gradient is addressed.

Inception also known as GoogLeNet [3], is based on the Hebbian principle and the intuition of multi-scale processing to increase the depth and width of a network.

Fig. 2 illustrates an example of an Inception module with three convolution layers and one max-pooling layer, there are three 1-by-1 layers which are used to change the dimension of the input to decrease the computational cost and another 1-by-1 layer acts as an identity transformation, same as in ResNet. The authors concatenate all feature maps as the next output to utilize image data in different scales. Due to the very deep structure, the authors add auxiliary classifiers connected to some intermediate layers to combat the vanishing gradient problem while providing regularization.

\section{ARCHITECTURE}

The idea of the proposed architecture is based on a simple intuition. When constructing a new convolution neural network, a proper convolution kernel size does affect dramatic improvement in the final result. For the standard design, we decrease the convolution kernel size in addition to decreasing the feature maps after the pooling process. For datasets like CIFAR or MNIST which contain images of small sizes, we often use 5-by-5 or 3-by-3 kernels. But based on my experience of designing networks, I found that with proper craft, convolution neural networks with a kernel of even sizes such as 4-by-4 or 2-by-2 have dramatically better performance.

Besides, for a certain size of feature maps, a multiple convolution network can learn different features under different scales which can maximize the usage of information for computer vision.

\section{A. Architecture Details}

The specific architecture is shown in Fig. 3. The numbers in brackets after a block name denote number of kernels in every convolution process. For the multiple convolution structure in Block-A, they are 1-by-1, 2-by-2, 3-by-3, 4-by-4 kernels respectively. The second convolution layer is 3-by-3 layers. Note that to decrease computational cost, we delete the 4-by4 layer in the last 6 layers of the multi-convolution layers, Although this modification does not have a significant teffect on test accuracy, it will boost the training speed by around $28 \%$.

Unlike the concatenating process in the Inception module, we use the maxout module [12] to join branches, this concept is shown in (2).

$$
h_{i}(x)=\max \left(z_{i j}\right)
$$

where $z_{i j}=x^{T} W_{\ldots i j}+b_{i j}$ and $W$ and $b$ are learned parameters, In a convolutional network, a maxout feature map can be constructed by taking the maximum across $\mathrm{k}$ affine feature maps. In our model, the maxout function is used to construct a competition process for feature maps.

In contrast to the Inception module that uses all feature maps as output, we think that the feature maps derived from different convolution layers have different weights; for instance, with respect to a large-sized input, large kernels have a better feature extraction ability comparing with smaller kernels, however after several down-samplings, smaller kernels perform better. With this competitive process, the network is expected to become self-optimizing with convolutions on different scales.

The activation function we use in experiments is the parametric rectified linear unit (PReLU) proposed in paper [32] which is defined as:

$$
f\left(y_{i}\right)=\left\{\begin{array}{cc}
y_{i}, & \text { if } y_{i}>0 \\
a_{i} y_{i}, & \text { if } y_{i} \leq 0
\end{array}\right.
$$

Here $y_{i}$ is the input of the nonlinear activation $f$ on the $i$ th channel, and $a_{i}$ is a learnable coefficient controlling the slope of the negative part. In contrast to ReLU, PReLU has negative values which reserve more trainable information, and allows a network to push the mean unit activations closer to zero, as in batch normalization, however PReLU has a lower computational complexity. Mean shifts toward zero speed up learning by bringing the normal gradient closer to the unit natural gradient, this occurs due to a reduced bias shift effect. Besides, PReLU can also obtain sparsity when trainable $a_{i}$ is small. 
We follow paper to initialize the standard deviation of weight, which is calculated as:

$$
\frac{1}{2} n_{l} \operatorname{Var}\left[w_{l}\right]=1
$$

where $w_{l}$ denotes the weights in $l$-th layer, and $n_{l}=k^{2} d$ denotes the entire connections between input and convolution neurons. For instance, for a 3-by-3 convolution layer with 256 kernels, we have $n_{l}=2304$. This leads to a zero-mean Gaussian distribution whose standard deviation (std) is $\sqrt{2 / n_{l}}$. As for standard deviation in fully connected layers, we set 0.01 for the first one and 0.001 for the last one.

For the forward propagation of the entire architecture, we have:

$$
X_{l+1}=\operatorname{PReLU}\left(\operatorname{conv}\left(f_{l}\left(X_{l}\right)\right)+i d\left(X_{l}\right)\right)
$$

where $\operatorname{conv}(\cdot)$ denotes the convolution process at the second convolution layer in block, $i d(\cdot)$ denotes identity mapping using the shortcut. Especially, we redefine $i d(\cdot)$ with a 1-by1 convolution to reduce the dimensions of $i d\left(x_{l}\right)$ to match those of $f_{l}\left(X_{l}\right)$ When the output dimensions do not match those of $\operatorname{conv}(\cdot), f_{l}(\cdot)$ denotes a multi-convolution process in $l$-th block defined by:

$$
\begin{aligned}
f_{l}\left(X_{l}\right)= & \sigma\left(W_{l 1}^{T} X_{i}+b_{l 1}, W_{l 2}^{T} X_{i}+b_{l 2},\right. \\
& \left.W_{l 3}^{T} X_{i}+b_{l 3}, W_{l 4}^{T} X_{i}+b_{l 4}\right)
\end{aligned}
$$

where $\sigma(\cdot)$ represents the maxout function, and the convolutional filters of the module are represented by the weight matrices $W_{l i}$ for $l \in\{1, \ldots, L\}$ and $i \in\{1,2,3,4\}$. Besides we use batch normalization after each convolution.

For back propagation, we have:

$$
\Delta \omega_{i}(t+1)=-\eta \frac{\partial E}{\partial \omega_{i}}+\alpha \Delta \omega_{i}(t)-\lambda \eta \omega_{i}
$$

where, $\eta$ represents the learning rate, $\alpha$ represents momentum, and $\lambda$ represents weight decay. And the momentum is set as 0.9 which is the same as that of $a_{i}$ in the PReLU activation function.

\section{B. Model Comparison}

At the beginning, we came up with three kinds of structures for multi-convolution layers as shown in Fig. 4. Comparing with Block A, Block B changes the position of the normal convolution layer and the multi-convolution layer, and block $\mathrm{C}$ uses two multi-convolution layers.

We also evaluate training errors of the three types of blocks on CIFAR-10 by training them for 500 epochs as Fig. 5 shows. The initial training error for block $\mathrm{C}$ is the largest because of additional parameters when comparing with Blocks $\mathrm{A}$ and $\mathrm{B}$. And block $\mathrm{A}$ has the fastest convergence speed among these three types and from the result in the next section, it is obvious that Block A has a better performance. For the reason that the architecture of Block A results the best performance, we hypothesize that we should not add activation function after the second convolution same as ResNet [4].

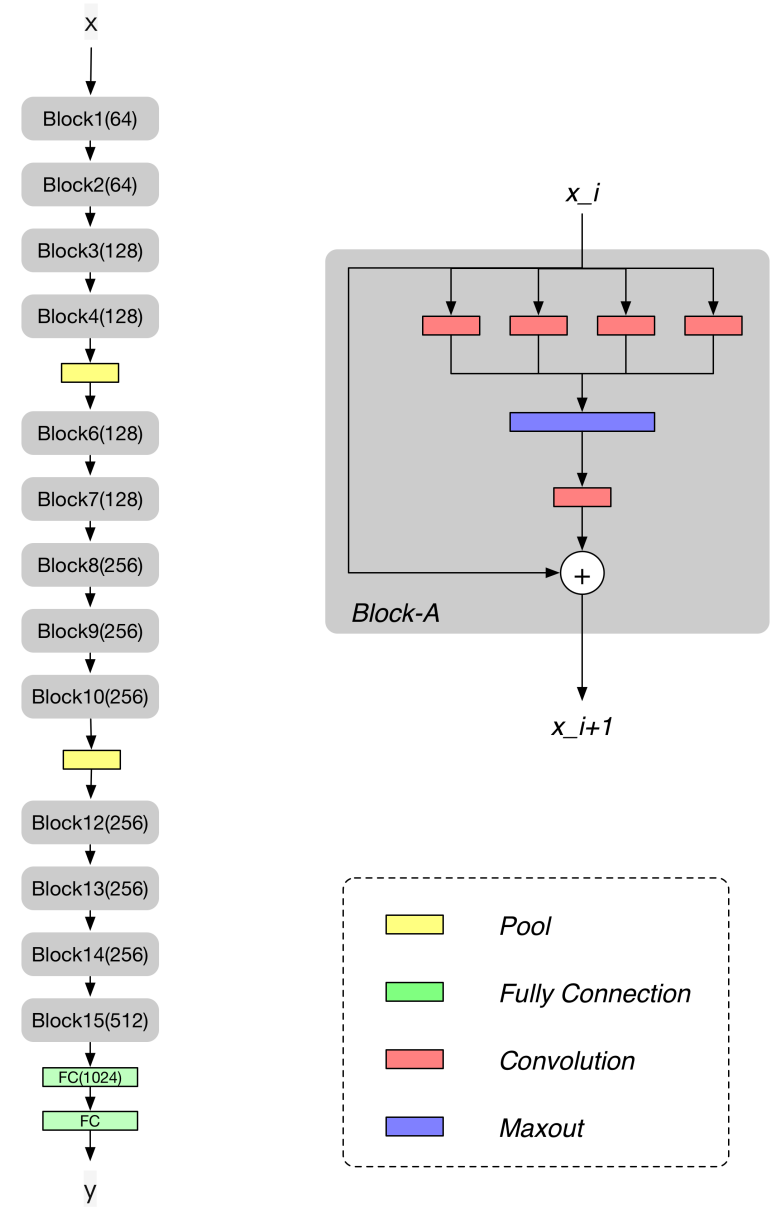

Fig. 3. Proposed architecture with Block-A.

\section{EXPERIMENTS}

We empirically demonstrate the effectiveness of the proposed method on a series of benchmark data sets, which are CIFAR-10, CIFAR-100, and MNIST. For all data sets we compare the results of our method with different layers and block structures, and other most competitive benchmarks.

In all experiments, weights are initialized obeying truncated normal distribution with a weight decay of 0.0001 for convolution layers and 0.004 for full-connected layers [4], and we use L2 regularization instead of a dropout network [27] to prevent overfitting. The mini-batch size for the experiment is 100 , initial learning rate is set as 0.1 and is divided by 10 when the error plateaus.

We use the GPU-accelerated TensorFlow framework with Python to perform the experiments specified in this paper. Our experimental environment is a desktop PC equipped with an i7-4790K CPU, 32GB memory and a 16GB GTX TITAN X graphic card.

\section{A. CIFAR-10}

CIFAR-10 [28] is a dataset which contains 32-by-32 images in RGB color space, in this dataset, objects are centered at the middle of the images with 10 categories of natural scene 

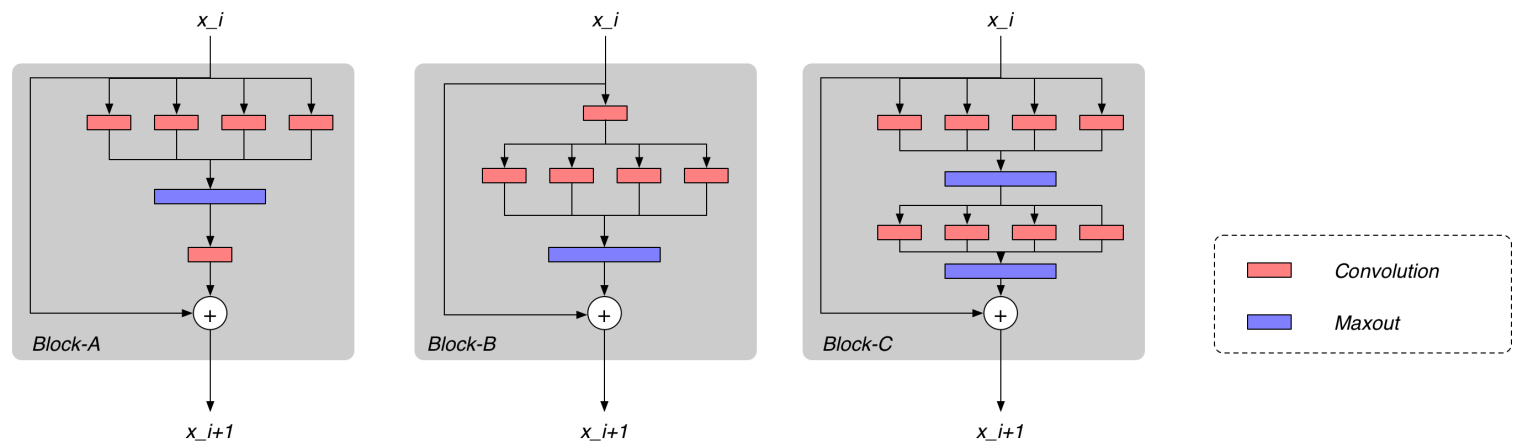

Fig. 4. Three kinds of multi-convolution blocks

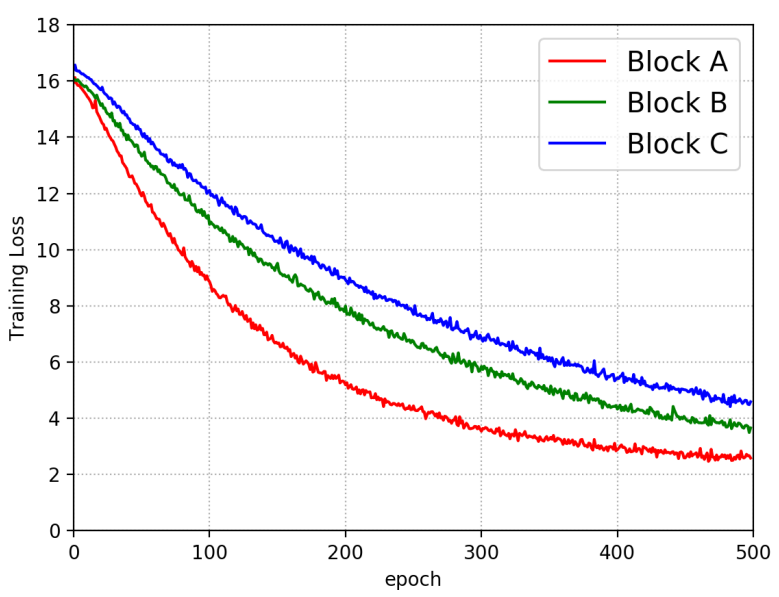

Fig. 5. Training loss on CIFAR-10 with three kinds of blocks.

objects. The training set and test set contain 50,000 and 10,000 images, respectively.

The result is shown in Table 1. A "+" in the name denotes standard data augmentation, which is Horizontal flipping and translation by 8 pixels from each image. Our method with type-A block and 26 layers in total achieves a $5.78 \%$ error rate with the CIFAR-10 dataset which is already a state-of-the-art result without resorting to massive data augmentation [33, 34] and has better performance comparing with other benchmarks. we simply add one block right before pooling layers and fully connected layer for 32-layer structure, it drastically reduces the error to $5.67 \%$.

Fig. 6 shows test errors on a 20-, 26-, 32-layer network with a type A block. Models are trained for 2000 epochs and test errors are recorded at every 100 epochs. Fig. 6 shows that the 32-layer model has the lowest test error and fastest convergence speed and the performance of the 26-layer model is the worst. We argue that this is because block construction is not optimized comparing with the 20-layer model, which also has less parameters and thus should converge faster at the beginning. On the other hand, the 32-layer model is more robust with respect to optimization.

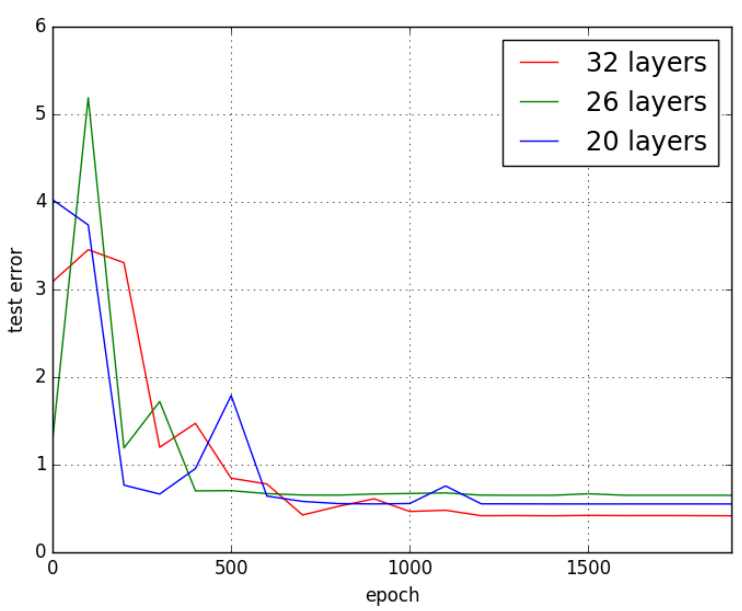

Fig. 6. Test error on CIFAR-10 with three different depths. All of these experiments consist of Block-A.

Besides, we tested type-B block and type-C block with 16 blocks, same as the analysis in Fig. 5. It was observed that performance of these two kinds of block was worse comparing with type-A block; nonetheless these blocks yielded a remarkable error rate. For this reason, we did not not conduct experiments on these two structures on the following dataset.

Also, ResNet with stochastic depth [21] achieved a 5.25\% error rate which is slightly better than our method; however since this model has 110 layers with around $1.7 \mathrm{M}$ parameters and three quarters of these are activated during the training, that is about $1.2 \mathrm{M}$ parameters, our method only has $36 \mathrm{k}$ parameters (32-layer model); hence we achieved a faster convergence speed. Besides, we have also trained these two models on our machine; it takes 19.6 hours (around 1.5 seconds per batch) for ResNet to converge and around 14.3 hours with respect to stochastic depth, but with our model consumes only 13.3 hours (around 0.8 second per batch) which is $7 \%$ faster comparing with stochastic depth. The details are shown in Table 2 and the training loss is shown in Fig. 7. 
TABLE I

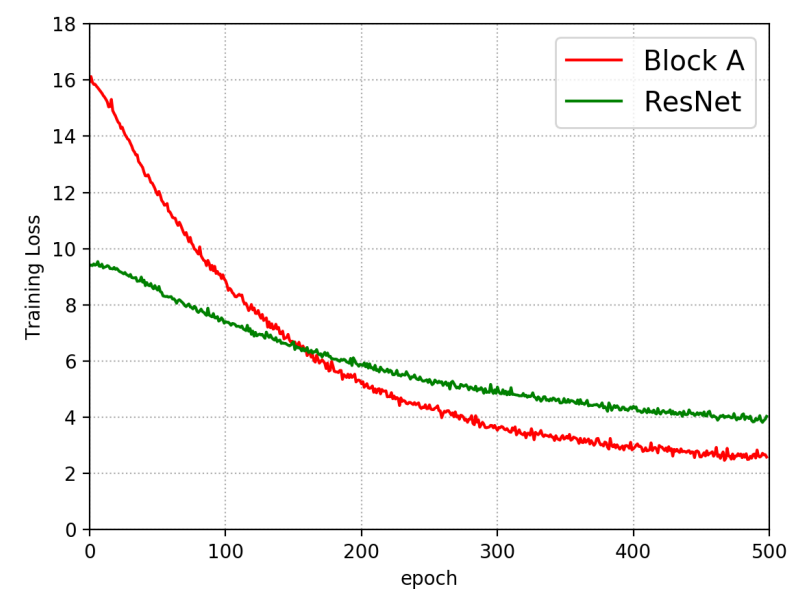

Fig. 7. Training loss on CIFAR-10.

\section{B. MNIST}

MNIST [29] dataset consists of 28-by-28 pixel grayscale images of handwritten digits from 0 to 9 , and is also a 10 classification problem, with 60,000 training and 10,000 test examples. The digits in these datasets have been sizenormalized and centered in a fixed-size image.

For the MNIST dataset, we did not augment dataset which is same as other methods. And we specially set the initial learning rate as 0.01 , which is slightly different to other experiments, because within learning rate of 0.1 , the model would diverge with a loss that equals $\mathrm{NaN}$.

Table 3 shows the error rate on the MNIST test set; our model with 32 layers achieves a $0.31 \%$ error rate which has the same performance as R-CNN [18] and Gen. Pool [20].

\section{CIFAR-100}

CIFAR-100 [28] is same as CIFAR-10 but there are 100 classes with only 600 images in each class; this dataset contains 500 and 100 images in its training dataset and test dataset respectively. Besides, this dataset has 20 coarse labels because some approaches have adopted a coarse-tofine training strategy to improve performance [30, 31]. But in our experiment, we only use fine labels with standard data augmentation.

The result is shown in Table 4. Our method yields a $25.26 \%$ error rate which is obviously better than $27.22 \%$ error rate of ResNet [4]. This result is slightly higher than that from stochastic depth [21] and ELU [26], but as we mentioned before, our model has less parameters and therefore is much faster.

\section{CONCLUSION}

Our experiments demonstrate clear advantages of multiple convolutional layers with residuals; these networks not only demonstrate computational efficiency but can also achieve state-of-the-art performance over several competitive benchmarks.
ERROR RATES (\%) ON CIFAR-10 TEST SET.

\begin{tabular}{c|c}
\hline Method & CIFAR10+ \\
\hline \hline Maxout [12] & 9.38 \\
DropConnect [13] & 9.32 \\
Net in Net [14] & 8.81 \\
Deeply Supervised [15] & 7.97 \\
All-CNN [16] & 7.25 \\
Learning Activation [17] & 7.51 \\
R-CNN [18] & 7.09 \\
Highway Network [19] & 7.60 \\
Gen. Pool [20] & 6.05 \\
Residual Network [4] & 6.41 \\
ResNet with stochastic depth [21] & 5.25 \\
\hline OURS-32 layers with A & $\mathbf{5 . 6 7}$ \\
OURS-26 layers with A & $\mathbf{5 . 7 8}$ \\
OURS-20 layers with A & 6.41 \\
OURS-32 layers with B & 6.90 \\
OURS-32 layers with C & 7.72 \\
\hline
\end{tabular}

TABLE II

COMPUTATIONAL COST IN CIFAR10+

\begin{tabular}{c|c|c|c}
\hline Method & Depth & Parameters & Training Time \\
\hline \hline Residual Network [4] & 110 & $1.7 \mathrm{M}$ & $19.6 \mathrm{~h}$ \\
\hline OURS-32 layers with $\mathbf{A}$ & $\mathbf{3 2}$ & $\mathbf{3 6 k}$ & $\mathbf{1 3 . 3 h}$ \\
\hline
\end{tabular}

TABLE III

ERROR RATES (\%) ON MNIST TEST SET.

\begin{tabular}{c|c}
\hline Method & MNIST \\
\hline \hline Stoch. Pooling [22] & 0.47 \\
Maxout [12] & 0.45 \\
DropConnect [13] & 0.57 \\
FitNet [23] & 0.51 \\
Net in Net [14] & 0.47 \\
DSN [15] & 0.39 \\
R-CNN [18] & 0.31 \\
Gen. Pool [20] & 0.31 \\
\hline OURS-32 layers with A & $\mathbf{0 . 3 1}$ \\
OURS-26 layers with A & 0.40 \\
\hline
\end{tabular}

TABLE IV

ERROR RATES (\%) ON CIFAR-100 TEST SET.

\begin{tabular}{c|c}
\hline Method & CIFAR100+ \\
\hline FitNet [23] & 35.04 \\
Deeply Supervised [15] & 34.57 \\
All-CNN [16] & 33.71 \\
R-CNN [18] & 31.75 \\
Highway Network [19] & 32.39 \\
MIM [24] & 29.20 \\
LSUV [25] & 27.66 \\
Residual Network [4] & 27.22 \\
Gen. Pool [20] & 32.37 \\
ResNet with stochastic depth [21] & 24.58 \\
ELU [26] & 24.28 \\
\hline OURS-32 layers with A & $\mathbf{2 5 . 2 6}$ \\
\hline
\end{tabular}

We believe that only increasing the depth is not enough to learn features in each layers and too many convolutional processes for a certain size of a feature map are not only computationally expensive but also not sparse. With an optimal wider architecture, the model converges image features in different scales more efficiently and has a significant quality 
gain at a modest increase of computational requirements compared to shallower and narrower architectures.

Comparing with ResNet [4] and ResNet with stochastic depth [21], our approach shows a better or just a little worse performance with only one third the depth and much lesser parameters, which yields solid evidence that moving to wider architectures is efficient and robust.

\section{REFERENCES}

[1] Krizhevsky, A, Hinton G. Learning multiple layers of features from tiny images. (2009).

[2] Simonyan, K, and Zisserman A. Very deep convolutional networks for large-scale image recognition. arXiv preprint arXiv:1409.1556 2014.

[3] Szegedy C, Liu W, Jia Y, Sermanet P, Reed S, Anguelov D, Erhan D, Vanhoucke V, Rabinovich A. Going deeper with convolutions. InProceedings of the IEEE conference on computer vision and pattern recognition 2015 (pp. 1-9)

[4] He K, Zhang X, Ren S, Sun J. Deep residual learning for image recognition. InProceedings of the IEEE conference on computer vision and pattern recognition 2016 (pp. 770-778).

[5] Hastad J, Goldmann M. On the power of small-depth threshold circuits. Computational Complexity. 1991 Jun 1;1(2):113-29.

[6] Ioffe S, Szegedy C. Batch normalization: Accelerating deep network training by reducing internal covariate shift. InInternational Conference on Machine Learning 2015 Jun 1 (pp. 448-456).

[7] Lee CY, Xie S, Gallagher P, Zhang Z, Tu Z. Deeply-supervised nets. InArtificial Intelligence and Statistics 2015 Feb 21 (pp. 562-570).

[8] Bengio Y, Lamblin P, Popovici D, Larochelle H. Greedy layer-wise training of deep networks. InAdvances in neural information processing systems 2007 (pp. 153-160).

[9] Glorot X, Bengio Y. Understanding the difficulty of training deep feedforward neural networks. InProceedings of the Thirteenth International Conference on Artificial Intelligence and Statistics 2010 Mar 31 (pp. 249-256).

[10] Erhan D, Bengio Y, Courville A, Manzagol PA, Vincent P, Bengio S. Why does unsupervised pre-training help deep learning? Journal of Machine Learning Research. 2010;11(Feb), pp.625-60.

[11] Nair V, Hinton GE. Rectified linear units improve restricted boltzmann machines. InProceedings of the 27th international conference on machine learning (ICML-10) 2010 (pp. 807-814).

[12] Goodfellow IJ, Warde-Farley D, Mirza M, Courville A, Bengio Y. Maxout networks. arXiv preprint arXiv:1302.4389. 2013 Feb 18.

[13] Wan L, Zeiler M, Zhang S, Le Cun Y, Fergus R. Regularization of neural networks using dropconnect. InInternational Conference on Machine Learning 2013 Feb 13 (pp. 1058-1066)

[14] Lin M, Chen Q, Yan S. Network in network. arXiv preprint arXiv:1312.4400. 2013 Dec 16.

[15] Lee CY, Xie S, Gallagher P, Zhang Z, Tu Z. Deeply-supervised nets InArtificial Intelligence and Statistics 2015 Feb 21 (pp. 562-570).

[16] Springenberg JT, Dosovitskiy A, Brox T, Riedmiller M. Striving for simplicity: The all convolutional net. arXiv preprint arXiv:1412.6806. 2014 Dec 21.

[17] Agostinelli F, Hoffman M, Sadowski P, Baldi P. Learning activation functions to improve deep neural networks. arXiv preprin arXiv:1412.6830. 2014 Dec 21.

[18] Liang M, Hu X. Recurrent convolutional neural network for object recognition. InProceedings of the IEEE Conference on Computer Vision and Pattern Recognition 2015 (pp. 3367-3375).

[19] Srivastava RK, Greff K, Schmidhuber J. Training very deep networks. InAdvances in neural information processing systems 2015 (pp. 23772385).

[20] Lee CY, Gallagher PW, Tu Z. Generalizing pooling functions in convolutional neural networks: Mixed, gated, and tree. InArtificial Intelligence and Statistics 2016 May 2 (pp. 464-472).

[21] Huang G, Sun Y, Liu Z, Sedra D, Weinberger KQ. Deep networks with stochastic depth. InEuropean Conference on Computer Vision 2016 Oct 8 (pp. 646-661). Springer International Publishing.

[22] Zeiler MD, Fergus R. Stochastic pooling for regularization of deep convolutional neural networks. arXiv preprint arXiv:1301.3557. 2013 Jan 16.
[23] Romero A, Ballas N, Kahou SE, Chassang A, Gatta C, Bengio Y. Fitnets: Hints for thin deep nets. arXiv preprint arXiv:1412.6550. 2014 Dec 19.

[24] Liao Z, Carneiro G. On the importance of normalisation layers in deep learning with piecewise linear activation units. InApplications of Computer Vision (WACV), 2016 IEEE Winter Conference on 2016 Mar 7 (pp. 1-8). IEEE

[25] Mishkin D, Matas J. All you need is a good init. arXiv preprint arXiv:1511.06422. 2015 Nov 19.

[26] Clevert DA, Unterthiner T, Hochreiter S. Fast and accurate deep network learning by exponential linear units (elus). arXiv preprint arXiv:1511.07289. 2015 Nov 23.

[27] Srivastava N, Hinton GE, Krizhevsky A, Sutskever I, Salakhutdinov R. Dropout: a simple way to prevent neural networks from overfitting. Journal of machine learning research. 2014 Jan 1;15(1):1929-58.

[28] Krizhevsky A, Hinton G. Learning multiple layers of features from tiny images. 2009.

[29] LeCun Y, Bottou L, Bengio Y, Haffner P. Gradient-based learning applied to document recognition. Proceedings of the IEEE. 1998 Nov;86(11):2278-324

[30] Dou H, Wu X. Coarse-to-fine trained multi-scale convolutional neural networks for image classification. InNeural Networks (IJCNN), 2015 International Joint Conference on 2015 Jul 12 (pp. 1-7). IEEE.

[31] Dutt A, Pellerin D, Quenot G. Improving Image Classification using Coarse and Fine Labels. InProceedings of the 2017 ACM on International Conference on Multimedia Retrieval 2017 Jun 6 (pp. 438-442). ACM.

[32] He K, Zhang X, Ren S, Sun J. Delving deep into rectifiers: Surpassing human-level performance on imagenet classification. InProceedings of the IEEE international conference on computer vision 2015 (pp. 1026-1034).

[33] Graham B. Fractional max-pooling. arXiv preprint arXiv:1412.6071. 2014 Dec 18.

[34] Springenberg JT, Dosovitskiy A, Brox T, Riedmiller M. Striving for simplicity: The all convolutional net. arXiv preprint arXiv:1412.6806. 2014 Dec 21 